\title{
A Longitudinal Study of High Scorers on the Hypomanic Personality Scale
}

By: Thomas R. Kwapil, Michael B. Miller, Michael C. Zinser, Loren J. Chapman, Jean Chapman and Mark Eckblad

Kwapil, T.R., Miller, M.B., Zinser, M.C., Chapman, L.J., Chapman, J., \& Eckblad, M. (2000). A longitudinal study of high scorers on the hypomanic personality scale. Journal of Abnormal Psychology, 109(2), 222-226. http://dx.doi.org/10.1037/0021-843X.109.2.222

\section{Made available courtesy of the American Psychological Association. The final published version can be found at http://www.apa.org/pubs/journals/abn/index.aspx.}

This article may not exactly replicate the final version published in the APA journal. It is not the copy of record. Figures and/or pictures may be missing from this format of the document.

\begin{abstract}
:
Former college students $(n=36)$ identified by high scores on the Hypomanic Personality Scale (HYP; Eckblad \& Chapman, 1986) were compared with control participants $(n=31)$ at a 13year follow-up assessment. As hypothesized, the HYP group reported more bipolar disorders and major depressive episodes than the control group. The HYP group also exceeded the control group on the severity of psychotic-like experiences, symptoms of borderline personality disorder, and rates of substance use disorders. HYP group members with elevated scores on the Impulsive-Nonconformity Scale (Chapman et al., 1984) experienced greater rates of bipolar mood disorders, poorer overall adjustment, and higher rates of arrest than the remaining HYP or control participants. (PsycINFO Database Record (c) 2012 APA, all rights reserved) (journal abstract)
\end{abstract}

Keywords: predictive validity of hypomanic personality scale | identification of those at risk for more severe mood psychopathology | former college students who scored high on hypomanic scale | 13 yr study | psychology

\section{Article:}


Acknowledgement: Thomas R. Kwapil is now at the Department of Psychology, University of North Carolina at Greensboro. Michael B. Miller is now at the Department of Psychology, University of Missouri. Michael C. Zinser is now at the Department of Psychology, University of Colorado at Denver.

This research was supported by grants from the National Institute of Mental Health and the Scottish Rite Schizophrenia Research Program. We are indebted to Nancy Sherwood for her assistance in interviewing participants and to Alina Sternberg and Denise Makuch for their assistance in tabulating data.

Correspondence concerning this article should be addressed to: Thomas R. Kwapil, Department of Psychology, University of North Carolina at Greensboro, P.O. Box 26164, Greensboro, North Carolina 27402-6164, Electronic Mail may be sent to: t_kwapil@uncg.edu.

The Diagnostic and Statistical Manual of Mental Disorders (fourth edition; DSM-IV;American Psychiatric Association, 1994) and the Research Diagnostic Criteria (RDC; Spitzer, Endicott, \& Robins, 1978) categorize hypomania as part of a mood disorder. However, researchers (e.g., Akiskal, 1992) have also characterized hypomania, or hyperthymia, as a personality style. Hypomania has frequently been reported as a premorbid characteristic in some bipolar patients. DSM-IV reports that $5 \%$ to $15 \%$ of patients experiencing hypomanic episodes will subsequently experience full-blown manic episodes, consistent with the finding from Akiskal, Khani, and Scott-Strauss (1979) that $6 \%$ of patients with cyclothymic personality developed manic episodes.

These findings suggest that psychometric identification of individuals with hypomanic personality characteristics might be useful for identifying those at risk for more severe mood psychopathology. Successful identification of such individuals should facilitate the study of the cause of such disorders and may hasten the development of treatment interventions that minimize the likelihood or severity of manic episodes. Identification of individuals with subclinical hypomanic features could be especially useful in family pedigree studies and for investigating biological and psychosocial factors that serve to precipitate or provide protection against the development of bipolar disorders.

\section{The Hypomanic Personality Scale (HYP)}

Eckblad and Chapman (1986) developed the HYP to identify individuals at risk for bipolar disorders and as a tool for resolving the heterogeneity of psychosis-prone samples. This selfreport scale contains 48 true-false items that inquire about features of hypomanic personality. The scale was constructed following the recommendations of Jackson (1970) for the development of personality measures. Sample items include the following: "Sometimes ideas and insights come to me so fast that I cannot express them all" (keyed true), and "I would rather be an ordinary success in life than a spectacular failure" (keyed false). The authors reported that 
the HYP Scale has a coefficient alpha reliability of .87 in an undergraduate sample $(n=1,519)$, and a test-retest reliability of .81 after an interval of 15 weeks $(n=89)$.

Eckblad and Chapman (1986) assessed the concurrent validity of the HYP Scale using diagnostic interviews with undergraduates who scored high on the scale $(n=40)$ and control participants ( $\mathrm{n}=40$ ). They found that $78 \%$ of the HYP group but none of the control group reported experiencing RDC hypomanic episodes. However, none of the participants reported a manic episode. The participants were subsequently rediagnosed by Thomas R. Kwapil using DSM-IV criteria (for the purposes of the present study), resulting in 21 (52\%) of the 40 original HYP participants meeting criteria for hypomanic episodes. On the basis of DSM-IV criteria, 25\% of the HYP group and none of the control participants qualified for a diagnosis of bipolar II disorder at the initial assessment, Fisher's exact test, $\mathrm{p}<.001$.

Eckblad and Chapman (1986) also reported that the HYP group exceeded the control group on several measures of hypomanic personality, including self-report of sociability, ambitiousness, speeded mood, increased energy, and perceived uniqueness, and had a greater proportion of participants who experienced depressive episodes. The HYP group also exceeded the control group on ratings of drug and alcohol use and severity of psychoticlike experiences and schizotypal symptoms.

Klein, Lewinsohn, and Seeley (1996) administered a subset of 15 items from the HYP Scale to participants in the Oregon Adolescent Depression Project. High scores on the abbreviated scale were associated with elevated rates of manic and depressive symptoms and substance use disorders. Petzel and Rado (1990) indicated that, unlike other measures that assess hypomania, the HYP Scale was not significantly correlated with measures of anxiety.

The present study continues the initial validation work of Eckblad and Chapman (1986) by examining the predictive validity of the HYP Scale in a 13-year longitudinal study of their original sample. The study investigates the functioning in early adulthood of individuals identified by the scale. Specifically, it explores whether these individuals are at heightened risk for (a) bipolar mood disorders, (b) major depressive episodes, (c) substance use disorders, (d) borderline personality features, (e) psychotic-like experiences, and (f) impaired functioning. These ratings represent primary or associated features of bipolar illnesses and are consistent with the measures used by Eckblad and Chapman (1986). On the basis of Akiskal's (1992) report that hyperthymic individuals often attain considerable professional achievement, we also examine whether HYP group members exceed control participants on measures of overall and vocational functioning. We also explore whether subgroups of HYP participants, defined by other personality characteristics or symptom clusters at the initial assessment, are at especially heightened risk for negative outcomes at the follow-up assessment.

\section{Method}

\section{Participants}




\section{Selection of participants and initial interview}

Participants in the present study were members of Eckblad and Chapman's (1986) sample. The HYP Scale was completed by approximately 1,500 undergraduates enrolled in an introductory psychology course at the University of Wisconsin in January 1982. Forty HYP participants (20 men and 20 women) were selected who had raw scores of 36 or higher on the scale (standard scores of 1.82 or higher for men and 1.67 or higher for women). Forty control participants (20 men, 20 women) were selected who scored less than 0.5 SD above the mean on the HYP Scale. The participants also completed the Magical Ideation Scale ( Eckblad \& Chapman, 1983), the Perceptual Aberration Scale ( Chapman, Chapman, \& Raulin, 1978), and the ImpulsiveNonconformity Scale ( Chapman et al., 1984). However, only scores on the HYP Scale were used for the selection of participants. The 80 participants received diagnostic interviews after their selection into the study (as described in Eckblad \& Chapman, 1986).

\section{Participants in the follow-up study}

Three original participants (all ethnic minorities) were dropped from the follow-up because reliable norms on the HYP Scale were only available for Caucasian students. We were able to reinterview 36 of 39 remaining HYP participants (92.3\%) and 31 of 38 remaining control group members (81.6\%). The reinterview rate did not differ significantly between the groups, Fisher's exact test, $\mathrm{p}=.14$. Of the 10 individuals who were not reinterviewed, 3 were not located, 1 died of cancer, and 6 declined to participate in the follow-up. Table 1 provides demographic information for those HYP and control group participants who were reinterviewed at the followup evaluation as well as their scores on the screening scales. 
Table 1

Demographic and Initial Testing Information for the HYP

and Control Groups

\begin{tabular}{|c|c|c|}
\hline Variable & $\begin{array}{c}\text { HYP } \\
(n=36)\end{array}$ & $\begin{array}{c}\text { Control } \\
(n=31)\end{array}$ \\
\hline \multicolumn{3}{|c|}{ Age at follow-up (years) } \\
\hline$M$ & $31.8^{* * *}$ & 33.6 \\
\hline$S D$ & 1.5 & 0.9 \\
\hline Male & $53 \%$ & $52 \%$ \\
\hline \multicolumn{3}{|l|}{ Father's social position } \\
\hline$M$ & 26.5 & 31.4 \\
\hline$S D$ & 15.2 & 17.8 \\
\hline \multicolumn{3}{|l|}{ Years to follow-up } \\
\hline M & $12.6^{*+6 *}$ & 14.2 \\
\hline$S D$ & 0.7 & 0.4 \\
\hline \multicolumn{3}{|c|}{ Perceptual Aberration Scale } \\
\hline$M$ & $0.63^{* * * *}$ & -0.41 \\
\hline$S D$ & 0.83 & 0.56 \\
\hline \multicolumn{3}{|l|}{ Magical Ideation Scale } \\
\hline$M$ & $0.85^{* * * *}$ & -0.52 \\
\hline$S D$ & 1.08 & 0.68 \\
\hline \multicolumn{3}{|c|}{ Impulsive-Nonconformity Scale } \\
\hline$M$ & $1.06 * * *$ & -0.21 \\
\hline$S D$ & 0.83 & 0.94 \\
\hline
\end{tabular}

\section{Materials and Procedures}

The follow-up interview consisted of a modified version of the Schedule for Affective Disorders and Schizophrenia—Lifetime Version (SADS-L; Spitzer \& Endicott, 1977) and portions of Loranger's (1988) Personality Disorder Examination (PDE) that assess schizotypal, schizoid, paranoid, and borderline personality disorders. The SADS-L was modified to obtain information required for DSM-IV diagnoses and information about psychoticlike experiences. The diagnostic interview assessed psychopathology and functioning since the time of the initial assessment. Participants were rated on the Hollingshead (1957) Two-Factor Index of Social Position and the Global Assessment Scale (GAS; Endicott, Spitzer, Fleiss, \& Cohen, 1976). Information was also obtained about family history of psychopathology. The Wisconsin Manual for Assessing Psychotic-like Experiences ( Chapman \& Chapman, 1980) was used to assess the degree of deviancy of psychotic-like and psychotic symptoms. We assessed psychotic-like experiences and the just-listed personality disorders at the follow-up based on Eckblad and Chapman's (1986) earlier finding of psychotic-like and schizotypal symptoms in the HYP group and because psychotic symptoms are not uncommon in manic episodes. All of the information was gathered from the participants' self-report. The HYP Scale was not readministered as part of the follow-up evaluation. 
The interviews lasted 1 to $4 \mathrm{hr}$ and were tape-recorded. The interviews were conducted by four psychologists who were experienced interviewers and diagnosticians. The interviewers had received extensive training with these specific interviews using tape-recorded samples of their work. The interviewers previously completed interviews and ratings of approximately 600 former college students as part of the longitudinal studies by Chapman et al. (1994) and Kwapil, Miller, Zinser, Chapman, and Chapman (1997). In addition, all of the personnel had extensive experience assessing patients with schizophrenia and severe mood disorders. A single rater, Thomas R. Kwapil, scored the diagnostic information, so interrater reliability was not available (the rater had administered and rated more than 500 structured diagnostic interviews to patient and nonpatient samples and taught a course on structured diagnostic interviewing). Previous studies by these researchers ( Kwapil, Chapman, Chapman, \& Miller, 1996) reported an intraclass correlation of .82 and kappa of .97 for ratings of psychotic-like experiences. The interviewers and rater were unaware of participants' group membership. Individuals were paid for their participation at the follow-up evaluation.

Standard $t$ tests were used to compare the groups when quantitative data were analyzed. The separate variance t statistic was calculated when the assumption of homogeneity of variance was violated. The Mann-Whitney U statistic was computed in one instance in which the distributions were highly skewed. Fisher's exact test was used when the data were categorical. The sampling procedures used in this study precluded the computation of reliable estimates of sensitivity and specificity.

\section{Results}

\section{Comparison of the HYP and Control Groups}

\section{Hypomanic symptoms}

Just as in the findings of Eckblad and Chapman (1986) at the initial assessment, the HYP group exceeded the control group on the percentage of participants who qualified for an episode of DSM-IV hypomania during the follow-up period (HYP $=28 \%$, control $=3 \%$; $<<.001$ ). All of these participants reported experiencing their most recent hypomanic episode within the past 2 years, ruling out the possibility that participants were simply describing episodes that they had previously reported at the initial assessment. The rates of hypomania did not differ significantly between HYP male (21\%) and HYP female (35\%) participants. Among the HYP participants who had reported hypomanic episodes at the initial interview, 33\% qualified for hypomanic episodes at the follow-up compared with $11 \%$ of the remaining HYP participants ( $\mathrm{p}=.11$ ). HYP group members who had reported hypomanic episodes at the initial interview (defined by DSMIV or RDC criteria) did not differ from the remaining HYP participants on measures of mood disorders or functioning at the follow-up assessment. The proportion of HYP participants reporting hypomanic episodes was lower at the follow-up than at the initial assessment ( $\mathrm{p}<.05$ ).

\section{Mood disorders}


Twenty-five percent of the HYP group and none of the control group qualified for bipolar disorders at the follow-up, $\mathrm{p}<.01$. Two HYP participants reported experiencing manic episodes, whereas none of the control group did so. Among the HYP group, 28\% suffered from unipolar depressive disorders compared with $19 \%$ of the control group (by definition, participants with unipolar depressive disorders did not suffer from manic or hypomanic episodes). Combining across unipolar and bipolar mood disorders, $36 \%$ of the HYP group and $10 \%$ of the control group experienced major depressive episodes during the follow-up, $\mathrm{p}<.05$. None of the participants experienced a psychotic illness. The groups did not differ on the rate of participants who reported a suicide attempt (HYP $=0 \%$, control $=3 \%$ ), nor on first- or second-degree relatives with depression $(\mathrm{HYP}=31 \%$, control $=26 \%)$ or mania $(\mathrm{HYP}=8 \%$, control $=6 \%)$.

\section{Psychiatric treatment}

The groups did not differ on the proportion of participants who reported receiving psychiatric treatment. None of the participants reported a history of psychiatric hospitalization, and the groups did not differ on the percentage of participants who reported receiving any outpatient psychotherapy (HYP = 36\%; control $=26 \%$ ). Treatment for mood disorders was sought by $25 \%$ of the HYP group and $10 \%$ of the control group, $\mathrm{p}=.09$. The groups did not differ on the percentage of participants who reported using antidepressant medication during the follow-up period (HYP = 14\%; control = 3\%). None of the participants had been prescribed moodstabilizing medications.

\section{Substance abuse}

Just as in Eckblad and Chapman's (1986) initial findings, the HYP group suffered significantly greater substance use and abuse than the control group at the follow-up assessment. Forty-four percent of the HYP group and 13\% of the control group qualified for DSM-IV substance use disorders during the follow-up period, $\mathrm{p}<.001$. The HYP group exceeded the control group on the rate of alcohol abuse/dependence ( $39 \%$ and $10 \%$, respectively; $\mathrm{p}<.001$ ) and the rate of marijuana abuse/dependence ( $19 \%$ and $3 \%$, respectively; $\mathrm{p}<.05$ ).

\section{Scholastic, occupational, and social functioning}

Table 2 summarizes comparisons between the HYP and control groups on measures of scholastic, occupational, and social functioning. The HYP group exceeded the control group on years of education and demonstrated a trend toward receiving a higher salary. However, they also demonstrated a trend toward a higher proportion of arrests. The distribution of income for the HYP was positively skewed because of the presence of 1 HYP participant with a very high income. In general, the types of occupations did not differ markedly between the groups. 
Table 2

Scholastic, Occupational, and Social Functioning at the Follow-Up Interview

\begin{tabular}{|c|c|c|}
\hline Variable & $\begin{array}{c}\text { HYP } \\
(n=36)\end{array}$ & $\begin{array}{c}\text { Control } \\
(n=31)\end{array}$ \\
\hline \multicolumn{3}{|l|}{ Global Assessment Scale } \\
\hline$M$ & 71.3 & 75.1 \\
\hline$S D$ & 11.7 & 8.4 \\
\hline \multicolumn{3}{|l|}{ Social position } \\
\hline$M$ & 21.7 & 24.0 \\
\hline$S D$ & 8.4 & 9.4 \\
\hline \multicolumn{3}{|l|}{ Years of education } \\
\hline$M$ & $17.0^{*}$ & 16.1 \\
\hline$S D$ & 1.9 & 1.8 \\
\hline Graduate or professional degree & $47 \% *$ & $23 \%$ \\
\hline Salary (thousands of dollars) & $45.0^{\text {*** }}$ & 40.0 \\
\hline Single (never married) & $36 \%$ & $25 \%$ \\
\hline Arrested & $25 \% * *$ & $10 \%$ \\
\hline \multicolumn{3}{|c|}{$\begin{array}{l}\text { Note. HYP }=\text { Hypomanic Personality Scale. High scores on the Global } \\
\text { Assessment Scale indicate superior overall functioning. Social position is } \\
\text { a weighted composite rating of educational and occupational functioning } \\
\text { for which higher scores indicate poorer accomplishment. Median values } \\
\text { are reported for salary. Means and standard deviations are reported for all } \\
\text { other quantitative variables. } \\
{ }_{p} p<.05 . * * p<.10 \text {. }\end{array}$} \\
\hline
\end{tabular}

\section{Psychoticlike experiences and personality disorder ratings}

Experiences of psychotic-like or psychotic deviancy were reported by $44 \%$ of the HYP group and $13 \%$ of the control group, $\mathrm{p}<.01$. The groups differed on the mean rating of such experiences (HYP M = 1.36, $\mathrm{SD}=1.69$; control $\mathrm{M}=0.48, \mathrm{SD}=1.31$ ), $\mathrm{t}(64)=2.39$, $\mathrm{p}<.05$. None of the HYP or control participants qualified for the full diagnosis of DSM-IV borderline, schizotypal, paranoid, or schizoid personality disorders. However, the HYP group exceeded the control group on PDE borderline dimensional score (HYP $\mathrm{M}=1.56$, $\mathrm{SD}=1.76$; control $\mathrm{M}=$ $0.19, \mathrm{SD}=0.79), \mathrm{t}(50)=4.17, \mathrm{p}<.001$, and demonstrated a trend toward higher ratings on schizotypal dimensional score (HYP $\mathrm{M}=1.19, \mathrm{SD}=1.64$; control $\mathrm{M}=0.58, \mathrm{SD}=1.36)$, $\mathrm{t}(65)$ = $1.68, \mathrm{p}=.10$.

\section{Comparison of Hypomanic Subgroups}

\section{Comparison of hypomanic and nonhypomanic HYP participants}

The 10 HYP group members who reported hypomanic episodes at the follow-up were compared with the remaining HYP participants on ratings of social position, GAS scores, drug and alcohol use, borderline and schizotypal personality dimensional scores, psychotic-like experiences, and income. The HYP participants with recent hypomanic episodes had significantly poorer GAS ratings ( $M=62.7, \mathrm{SD}=7.3$ ) than the remaining HYP participants ( $\mathrm{M}=74.6, \mathrm{SD}=11.5)$, $\mathrm{t}(26)$ $=3.69, \mathrm{p}<.01$. 


\section{Potentiation of hypomanic traits by impulsive nonconformity}

HYP participants who had standard scores of 1.5 or higher on the Impulsive-Nonconformity Scale $(n=9)$ exceeded the remaining HYP participants $(n=27)$ at the follow-up assessment on the rate of bipolar mood disorders (67\% to $11 \%$, respectively; $\mathrm{p}<.001$ ). Twenty-two percent of the HYP/impulsive-nonconformity individuals reported manic episodes at the follow-up, compared with none of the remaining HYP individuals (Fisher's exact test $=.06$ ). The HYP/impulsive-nonconformity subgroup also exceeded the remaining HYP individuals on proportion of participants arrested (56\%-15\%, $\mathrm{p}<.05)$, borderline dimensional score $(\mathrm{p}<.05)$, and alcohol use $(\mathrm{p}<.05)$. The HYP/impulsive-nonconformity subgroup also had significantly poorer overall functioning, as measured by the GAS $(\mathrm{p}<.05)$, than the control group. Analogous comparisons of HYP subgroups based on the Perceptual Aberration and Magical Ideation Scales did not yield significant differences. Neither impulsive-nonconformity nor perceptual aberration/magical ideation moderated the relationship between HYP score and social position, income, or education.

\section{Discussion}

The results indicate that individuals with hypomanic personality, identified in late adolescence or early adulthood by elevated scores on the HYP Scale, are at a heightened risk for DSM-IV bipolar disorders in adulthood, although the proportion of HYP participants reporting hypomanic episodes was less at the follow-up assessment than at the initial interview. Interestingly, the HYP participants who had hypomanic episodes at the initial assessment did not demonstrate a markedly higher rate of hypomanic episodes at the follow-up compared with the remaining HYP group members. It may be that the scale identifies a personality style that does not always manifest as recurring hypomania.

The HYP group did not appear to be at especially heightened risk for manic episodes at the follow-up assessment. However, the rate of manic episodes (6\%) was consistent with the lifetime rates of manic episodes reported for individuals with hypomania ( American Psychiatric Association, 1994). Furthermore, our participants still have a significant period of risk remaining for developing manic episodes. Our selection of high-risk participants from a college sample may delay the initial development of manic episodes.

The HYP group exceeded the control group on the proportion of participants who reported major depressive episodes at follow-up. Consistent with findings from Eckblad and Chapman's (1986) initial assessment and from Klein et al.'s (1996) use of an abbreviated version of the HYP Scale, the HYP group had significantly higher rates of substance use disorders. The heightened rate of depression and substance use problems in the HYP group is striking because both are associated features of hypomanic and manic episodes, but neither are inquired about by the HYP Scale. Contrary to findings by Klein et al. (1996), the groups did not differ on proportion of participants who had attempted suicide. 
A clear pattern of differences did not emerge when the HYP and control groups were compared on measures of overall functioning. The HYP group contained a greater proportion of participants who had received graduate degrees and showed a trend toward attaining a higher salary. However, they also showed a trend toward more arrests. Consistent with descriptions of hyperthymic individuals ( Arieti, 1974; Fieve \& Dunner, 1975), these individuals appeared to be relatively successful but heedless of social conventions.

The heterogeneity of the HYP group, with regard to overall functioning, spurred further investigation of deviant subgroups within the HYP group. The HYP participants who had initially endorsed impulsive, antisocial traits, as measured by elevated scores on the ImpulsiveNonconformity Scale, reported higher rates of bipolar disorder and arrests and more severe ratings of alcohol use and borderline personality symptoms than the remaining HYP participants. This is consistent with reports by Klein et al. (1996), who found that a history of disruptive behaviors was correlated with a history of mood disorders among adolescents with hypomanic traits. The combination of hypomanic personality and nonconforming traits apparently predisposes individuals to especially poor outcomes. The finding at follow-up of an especially heightened rate of bipolar disorder in the HYP/Impulsive-Nonconformity participants appears to be due to a combination of the two scales, not solely to the Impulsive-Nonconformity Scale. Chapman, Chapman, Kwapil, Eckblad, and Zinser (1994) found that participants identified by deviantly high scores on the Impulsive-Nonconformity Scale did not report elevated rates of manic or hypomanic episodes than control participants at a 10-year follow-up.

That the HYP group exceeded the control group on ratings of borderline personality traits is not surprising, given that affective dysregulation is a central feature of both hypomania and borderline personality disorder (e.g., Akiskal, 1992; Gunderson \& Singer, 1975). Akiskal (1981) discussed the fact that similarity in symptoms causes hypomania to be confused with borderline character pathology. Our findings are consistent with the DSM-IV report that borderline personality features are often comorbid with mood disorders. However, high scorers on the HYP Scale did not develop borderline personality disorder, despite their heightened rates of bipolar disorder.

Although we have interpreted our findings as indicating that the HYP Scale predicts risk for bipolar disorders, a competing explanation is that the scale more generally measures affective dysregulation. Affective dysregulation is viewed as a feature of numerous conditions, including mood disorders, externalizing disorders, and borderline personality disorder. However, affective dysregulation is not always operationalized consistently across domains. The HYP Scale appears to identify individuals who display affective, cognitive, and behavioral dysregulation consistent with the symptoms of bipolar disorders. However, the present study did not specifically investigate hypotheses related to affective dysregulation.

The present study examined the predictive validity of the HYP Scale in predominately middleclass, Caucasian college students. This raises concerns about the generalizability of the findings 
to more ethnically and socially diverse samples. The Epidemiological Catchment Area program ( Robins, 1984) reported that noncollege graduates have twice the risk of experiencing manic episodes than do college graduates. College students tend to be intellectually and socially advantaged, factors that might provide these individuals with protection against negative outcomes. The use of the scale with high-functioning samples may underestimate the predictive validity of the measure.

Although additional validation studies with socially and ethnically diverse samples are needed, the present findings suggested several promising research applications for the HYP Scale. The identification of individuals who endorse hypomanic characteristics may be useful for understanding the relationship between hyperthymia and bipolar disorders. The scale may also improve the precision of family pedigree studies of bipolar disorder by providing a method for identifying individuals with subclinical bipolar features. Furthermore, the study of such individuals may facilitate the identification of biological and psychosocial factors that serve to precipitate or provide protection against the development of bipolar disorders. The findings do not support clinical uses of the HYP Scale at this time. Furthermore, early intervention based on HYP scores does not appear warranted because most of the HYP participants had not developed manic episodes at the follow-up. However, two thirds of the HYP/Impulsive-Nonconformity participants developed bipolar disorders at the follow-up (and one third of those individuals developed manic episodes). These findings encourage further study to identify those HYP participants who are at especially heightened risk for ongoing hypomanic episodes and the development of manic episodes.

\section{References:}

Akiskal, H. S. ( 1981). Subaffective disorders: Dysthymic, cyclothymic and bipolar II disorders in the "borderline" realm. Psychiatric Clinics of North America, 4, 25- 46.

Akiskal, H. S. ( 1992). Delineating irri table and hyperthymic variants of the cyclothymic temperament. Journal of Personality Disorders, 6, 326- 342.

Akiskal, H. S., Khani, M. K., \& Scott-Strauss, A. ( 1979). Cyclothymic temperamental disorders. Affective Disorders: Special Clinical Forms, 2, 527- 554.

American Psychiatric Association. ( 1994). Diagnostic and statistical manual of mental disorders (4th ed.). Washington, DC: Author.

Arieti, S. ( 1974). Interpretation of schizophrenia ( 2nd ed.). New York: Basic Books.

Chapman, L. J., \& Chapman, J. P. ( 1980). Scales for rating psychotic and psychotic-like experiences as continua. Schizophrenia Bulletin, 6, 476- 489. 
Chapman, L. J., Chapman, J., Kwapil, T., Eckblad, M., \& Zinser, M. C. ( 1994). Putatively psychosis-prone subjects 10 years later. Journal of Abnormal Psychology, 103, 171- 183.

Chapman, L. J., Chapman, J. P., Numbers, J. S., Edell, W. S., Carpenter, B. N., \& Beckfield, D. ( 1984). Impulsive nonconformity as a trait contributing to the prediction of psychotic-like and schizotypal symptoms. Journal of Nervous and Mental Disease, 172, 681- 691.

Chapman, L. J., Chapman, J. P., \& Raulin, M. L. ( 1978). Body-image aberration in schizophrenia. Journal of Abnormal Psychology, 87, 399- 407.

Eckblad, M., \& Chapman, L. J. ( 1983). Magical ideation as an indicator of schizotypy. Journal of Consulting and Clinical Psychology, 51, 215- 225.

Eckblad, M., \& Chapman, L. J. ( 1986). Development and validation of a scale for hypomanic personality. Journal of Abnormal Psychology, 95, 214- 222.

Endicott, J., Spitzer, R. L., Fleiss, J. L., \& Cohen, J. ( 1976). The global assessment scale: A procedure for measuring overall severity of psychiatric disturbance. Archives of General Psychiatry, 33, 766- 771.

Fieve, R. R., \& Dunner, D. L. ( 1975). Unipolar and bipolar affective states: An alternative proposal. In F.Flach \& S.Draghi (Eds.), The Nature and treatment of depressionNew York: Wiley.

Gunderson, J. G., \& Singer, M. T. ( 1975). Defining borderline patients: An overview. American Journal of Psychiatry, 132, 1- 10.

Hollingshead, A. B. ( 1957). Two-Factor Index of Social Position Unpublished manuscript, Department of Psychology, Yale University.

Jackson, D. N. ( 1970). A sequential system for personality scale development. In C.

N.Spielberger (Ed.), Current topics in clinical and community psychology ( Vol. 2, (pp. 61- 96). New York: Academic Press.

Klein, D. N., Lewinsohn, P. M., \& Seeley, J. R. ( 1996). Hypomanic personality traits in a community sample of adolescents. Journal of Affective Disorders, 38, 135- 143.

Kwapil, T. R., Chapman, L. J., Chapman, J. P., \& Miller, M. B. ( 1996). Deviant olfactory experiences as indicators of risk for psychosis. Schizophrenia Bulletin, 22, 371- 382.

Kwapil, T. R., Miller, M. B., Zinser, M. C., Chapman, J. P., \& Chapman, L. J. ( 1997). Magical ideation and social anhedonia as predictors of psychosis proneness: A partial replication. Journal of Abnormal Psychology, 106, 491- 495. 
Loranger, A. W. ( 1988). Personality disorder examination (PDE) manual. Yonkers, NY: DV Communications.

Petzel, T. P., \& Rado, E. D. ( 1990). Divergent validity evidence for Eckblad and Chapman's Hypomanic Personality Scale. Journal of Clinical Psychology, 46, 43- 46.

Robins, L. N. ( 1984). Lifetime prevalence of specific psychiatric disorders in three sites. Archives of General Psychiatry, 41, 949- 958.

Spitzer, R. L., \& Endicott, J. ( 1977). Schedule for affective disorders and schizophrenia-lifetime version (SADS-L). New York: New York State Psychiatric Institute.

Spitzer, R. L., Endicott, J., \& Robins, E. ( 1978). Research diagnostic criteria (RDC) for a selected group of functional disorders ( 3rd ed.). New York: State Psychiatric Institute. 